TI 2001-043/2

Tinbergen Institute Discussion Paper

Reducing the Dimensionality

of Linear Quadratic Control

Problems

Ronald J. Balvers',2

Douglas W. Mitchell

${ }^{\top}$ West Virginia University, ${ }^{2}$ Tinbergen Institute 
Tinbergen Institute

The Tinbergen Institute is the institute for economic research of the Erasmus Universiteit Rotterdam, Universiteit van Amsterdam and

Vrije Universiteit Amsterdam.

Tinbergen I nstitute Amsterdam

Keizersgracht 482

1017 EG Amsterdam

The Netherlands

Tel.: +31.(0)20.5513500

Fax: $\quad+31 .(0) 20.5513555$

Tinbergen Institute Rotterdam

Burg. Oudlaan 50

3062 PA Rotterdam

The Netherlands

Tel.: $\quad+31 .(0) 10.4088900$

Fax: $\quad+31 .(0) 10.4089031$

Most TI discussion papers can be downloaded at

http://www.tinbergen.nl 


\title{
Reducing the Dimensionality of Linear Quadratic Control Problems*
}

\author{
February 2001
}

Ronald J. Balvers

Department of Economics

P.O. Box 6025

West Virginia University

Morgantown, WV 26506-6025

Phone: (304) 293-7880

Email: rbalvers@wvu.edu
Douglas W. Mitchell

Department of Economics

P.O. Box 6025

West Virginia University

Morgantown, WV 26506-6025

Phone: (304) 293-7868

Email: dmitchel@wvu.edu

\begin{abstract}
In linear-quadratic control (LQC) problems with singular control cost matrix and/or singular transition matrix, we derive a reduction of the dimension of the Riccati matrix, simplifying iteration and solution. Employing a novel transformation, we show that, under a certain rank condition, the matrix of optimal feedback coefficients is linear in the reduced Riccati matrix. For a substantive class of problems, our technique permits scalar iteration, leading to simple analytical solution. By duality the technique can also be applied to Kalman filtering problems with a singular measurement error covariance matrix.

JEL classification: C61; C63, D83
\end{abstract}

Keywords: Linear-quadratic control; Riccati equation; Riccati reduction; Kalman filtering; Intertemporal optimization

* The authors thank Victor Claar for stimulating their interest in the subject material. 


\section{Reducing the Dimensionality of Linear Quadratic Control Problems}

\section{Introduction}

The preeminence of computable general equilibrium models has stimulated interest in the solution procedures for larger-scale models. Most commonly, linear rational expectations models are considered which, typically, are derivable from linear-quadratic control (LQC) problems. The recent work by Sims (2000), Binder and Pesaran (2000), King and Watson (1997, 1998), Amman (1997), Amman and Neudecker (1997), Anderson et al. (1997), Anderson and Moore (1985), Ehlgen (1999), and Klein (2000) concentrates on numerical procedures that (1) allow speedy and convenient computation of results, and (2) apply as generally as possible, in particular to systems with non-invertibilities stemming from a singular transition matrix or a singular control cost matrix. Generally, objective (2) has been attained at the expense of increasing the dimensionality of the system. These papers improve on the work of Vaughan (1970) and Blanchard and Kahn (1980).

The focus in the current paper is on simplifying the system when singularities occur.

These simplifications lead to very simple analytical solutions in a substantial class of problems and in computationally efficient methods in all other cases, for finite or infinite horizon. We obtain a simple rank expression that places an upper bound on the effective dimensionality of the system for analytical and computational purposes: Prior computation of the rank of a composite matrix constructed from all coefficient matrices in the problem statement allows the researcher to establish this bound. The advantage is that one may readily determine up front whether the system has a simple explicit solution, or to what extent reformulation of the problem along the lines delineated here may reduce computation time or improve the transparency of the model.

The approach developed in this paper is aided by the duality between linear-quadratic 
control and Kalman filtering as first described by Kalman (1960). A key feature of our approach is the use of the covariance matrices of Kalman filtering and their inverses (the latter being related to the Bayesian concept of precision). The Kalman-updated covariance matrix plays an important role that is not typically exploited in LQC analyses.

The Kalman approach also provides intuition for why the dimensionality of a particular system may be reduced. Consider the following class of Kalman problems, which is exemplified by Claar's (2000) model of the cyclical and natural unemployment rates. An observation depends linearly on two unobserved state variables following stochastic processes: $w_{t}=y_{1 t}+y_{2 t}$. One may describe the uncertainty of the state by considering the conditional variances $\sigma_{1 t}^{2}, \sigma_{2 t}^{2}$ of the state variables and their conditional covariance $\sigma_{12 t}=\sigma_{21 t}$ (three numbers, stored in a $2 \times 2$ covariance matrix). However, conditional on having observed $w_{t}$, it is easy to derive that $\sigma_{1 t}^{2}=\sigma_{2 t}^{2}=-\sigma_{12 t}$ so that one number is sufficient to describe the state uncertainty. This simplification was employed by Balvers and Cosimano (1994) in reducing the dimensionality of their active learning model, but the approach has not been systematically investigated. While the intuition for simplification here is straightforward, our rank expression implies a potentially complex interaction between the different singularities in the system that is not always intuitive.

Mitchell (2000) derived explicit analytical solutions to the $2 \times 1$ linear-quadratic control problem (two target variables and one uncosted control in the control case, or two state variables and one perfect observation in the Kalman case), but his results were not obviously generalizable. In this paper we significantly extend the class of linear-quadratic models which can be simplified or solved analytically in simple fashion.

The paper is organized as follows. Section 2 contains the theoretical approach in which 
we derive theorems that state how the dimensionality of the model can be reduced and by how much, how under a certain rank condition the optimal feedback control matrix is linearly related to the reduced Riccati matrix (allowing a simple time-invariant linear transformation to obtain the feedback matrix once the appropriate reduced Riccati matrix is found), and how the reduced Riccati iterations can be replaced by linear iteration of a related matrix. Section 3 discusses implications for applied optimal control and Kalman filtering and presents some control and Kalman examples. In Section 4 we conclude the paper by providing a "how to" summary of our technique for practical use.

\section{THEORY}

\subsection{The control problem}

In this section we show how to reduce the dimension of the Riccati equation of optimal control, without assuming the presence of an invertible control cost matrix or invertibility of the transition matrix. In so doing we illuminate the underlying structure of the dynamics. The two initial lemmas establish the structure of the Riccati matrices, and Theorem 1 gives the reduced dynamics. The reduced problem is shown in Theorem 2 to be sometimes amenable to further simplification of the solution for the control feedback matrix. Then Theorem 3 shows how to obtain the Riccati sequence by linear iteration of a related matrix, permitting speedy calculation of the control sequence or its steady state. Theorems $1^{*}$ and $3 *$ deal with a further reduction of the Riccati matrix dimension which is possible under some conditions.

The reduction that we present is unrelated to the concept of reducing a system to "minimal" form in the sense of obtaining the lowest state vector dimension necessary for optimal Kalman filtering. A system is in minimal form if it is both controllable and observable (see Hannan and Deistler, 1988; note that some authors, especially in the Kalman filtering context, use 
terminology different from ours, which is typically used in the control literature). In our control context, we assume that the state cost matrix is nonsingular, so that minimality is equivalent to controllability. Our Riccati reduction applies even if the system is controllable and hence minimal. This reduction can be achieved because the effective dimension of the Riccati matrix is less than the dimension of the state vector even if the latter is minimal.

A typical statement of the optimal control problem is:

$$
\begin{aligned}
& V\left(y_{s}, s\right)=\begin{array}{l}
\operatorname{Min} \\
\left\{u_{t}\right\}_{s+1}^{T}
\end{array}\left(1 / 2\left(y_{T}^{\prime} K_{T} y_{T}\right)+\sum_{t=s+1}^{T-1}\left[1 / 2\left(y_{t}^{\prime} K y_{t}\right)\right]\right), \\
& \text { subject to } y_{t}=A y_{t-1}+C u_{t}, \quad t=s+1, \ldots, T, \quad y_{s} \text { given, }
\end{aligned}
$$

where the state cost matrices $K$ and $K_{T}$, and the transition matrix $A$ are $n \times n$, the control multiplier matrix $C$ is $n x k$, the state vector $y_{t}$ is $n x 1$, and the control vector $u_{t}$ is $k x 1$. The cost matrix $K$ and the terminal cost matrix $K_{T}$ are positive definite, and $C$ has full column rank; $A$ need not have full rank. If the original problem statement has control costs, one can augment the state vector with the costed controls [see Chow (1975)], putting all costs on the state vector and thus giving the problem formulation in equations (1). ${ }^{1}$ It is well known [Chow (1975)] that the optimal controls are given by:

${ }^{1}$ In this case the reduction in this paper constitutes a net reduction of the Riccati matrix dimension if the original control cost matrix has less than full rank (or, even if not, often if the transition matrix has less than full rank).

Discounting and additive uncertainty in the state equation can easily be incorporated by redefining the transition matrix and the state and control vectors, and appealing to certainty equivalence, respectively. In addition, if the original problem statement has cross-product costs between the state vector and the control vector, these can be removed by redefinition of the state cost matrix and the control vector [see for instance Ljungqvist and Sargent (2000), p. 640]. 


$$
\begin{aligned}
& u_{t}^{o p t}=-\left(C^{\prime} H_{t} C\right)^{-1} C^{\prime} H_{t} A y_{t-1} \equiv-F_{t} y_{t-1}, \quad t \leq T, \\
& H_{t-1}=K+A^{\prime} H_{t} A-A^{\prime} H_{t} C\left(C^{\prime} H_{t} C\right)^{-1} C^{\prime} H_{t} A, \quad H_{T}=K_{T}, \quad t \leq T,
\end{aligned}
$$

where the symmetric $n \times n$ matrix $H_{t}$ is positive definite.

\subsection{The basic reduction}

Equation (3) can be written as equations (4) and (5):

$$
\begin{aligned}
& H_{t-1}=K+A^{\prime} P_{t} A, \quad t \leq T, \\
& P_{t}=H_{t}-H_{t} C\left(C^{\prime} H_{t} C\right)^{-1} C^{\prime} H_{t}, \quad t \leq T .
\end{aligned}
$$

The symmetric $n \times n \quad P_{t}$ matrix is not typically employed in dealing with optimal control problems, but in the dual Kalman filtering context has the familiar interpretation of the covariance matrix for the unobserved state variable for the current period conditional on current information (while $H_{t}$ is the covariance matrix conditional on the previous period's information).

The approach in this paper is to exploit restrictions inherent in the $P_{t}$ matrix to simplify the solution of problem (1). By equation (5) we have:

(6) $P_{t} C \equiv\left[\begin{array}{ll}P_{1 t} & P_{2 t} \\ P_{3 t} & P_{4 t}\end{array}\right]\left[\begin{array}{l}C_{1} \\ C_{2}\end{array}\right]=0_{n, k}, \quad t \leq T$, 
where, defining $q=n-k$, we have that $P_{1 t}$ is $q x q, P_{2 t}$ is $q x k, P_{3 t}=P_{2 t}^{\prime}$, and $P_{4 t}$ is $k x k$;

$C_{1}$ is $q x k$, and $C_{2}$ is $k x k . \quad P_{1 t}$ and $P_{4 t}$ are symmetric.

Since $C$ is of full column rank, there is at least one $k x k$ sub-matrix of $C$ that is invertible. Proper prior arrangement of the $y_{t}$ vector (and concomitant arrangement of $C, A, K$, and $K_{T}$ ) is thus sufficient to guarantee that $C_{2}$ is invertible. We can then derive:

LEMMA 1 (REDUCTION TO THE DYNAMIC CORE OF $P_{t}$ ). The $n x n$ matrix $P_{t}$ can be written as:

$$
\begin{aligned}
& P_{t}=M \Phi_{t} M^{\prime}, \quad \Phi_{t} \equiv P_{1 t}, \quad t \leq T \\
& M \equiv\left[\begin{array}{c}
I_{n-k} \\
-\left(C_{2}^{\prime}\right)^{-1} C_{1}^{\prime}
\end{array}\right],
\end{aligned}
$$

where $M$ is an $n \times q$ matrix, and $\Phi_{t}$ is invertible with dimensions $q \times q$.

Proof. From equations (6) it is straightforward to relate $P_{3 t}\left(=P_{2 t}^{\prime}\right)$ and $P_{4 t}$ to $P_{1 t}$. The first equation in (6) gives $P_{2 t}=-P_{1 t} C_{1} C_{2}^{-1}$. Transpose (to produce $P_{3 t}$ ) and substitute into the second equation (noting the symmetry of $P_{1 t}$ as follows from the symmetry of $P_{t}$ ) which yields $P_{4 t}=\left(C_{2}^{\prime}\right)^{-1} C_{1}^{\prime} P_{1 t} C_{1} C_{2}^{-1}$. Then factor out the $M$ and $M^{\prime}$ matrices to produce equation (7). To show that $\Phi_{t}$ is invertible, note from equation (5) that $P_{t}$ can be written as the product 
$H_{t}\left[I_{n}-C\left(C^{\prime} H_{t} C\right)^{-1} C^{\prime} H_{t}\right]$, where the matrix in brackets is idempotent with trace equal to $\operatorname{trace}\left(I_{n}\right)-\operatorname{trace}\left(I_{k}\right)$ and thus rank $n-k=q$. Thus, since $H_{t}$ has full rank $n, P_{t}$ has rank $q{ }^{2}$

Equation (7) then implies that $\operatorname{rank}\left(\Phi_{t}\right) \geq q$, and since $\Phi_{t}$ has dimension $q$ it must have full rank.

It will be important to relate $\Phi_{t}$ to the solution of problem (1) - equations (2) and (3) in a meaningful way. Lemma 2 provides a useful link.

LEMMA 2 (RELATING $\Phi_{t}$ AND $\left.H_{t}\right)$. The $q x q$ matrix $\Phi_{t}$ in equation (7) is positive definite and is given by:

$$
\Phi_{t}=\left(M^{\prime} H_{t}^{-1} M\right)^{-1}, \quad t \leq T .
$$

Proof. Post-multiply equation (5) by $H_{t}^{-1} P_{t}$ and then use the transpose of equation (6). This yields $P_{t}=P_{t} H_{t}^{-1} P_{t}$, so that, interestingly, $H_{t}^{-1}$ is seen to be a generalized inverse of $P_{t}$. Next use equation (7) in the right-hand side of this equation and pre-multiply by $\left[\begin{array}{ll}I_{q} & 0\end{array}\right]$ and post-multiply by $\left[\begin{array}{ll}I_{q} & 0\end{array}\right]^{\prime}$ to pick out the upper left block $P_{1 t} \equiv \Phi_{t}$ of the matrix, yielding:

$$
\Phi_{t}=\left[I_{q} 0\right] M \Phi_{t} M^{\prime} H_{t}^{-1} M \Phi_{t} M^{\prime}\left[\begin{array}{c}
I_{q} \\
0
\end{array}\right], \quad t \leq T .
$$

\footnotetext{
${ }^{2} P_{t}$ has rank $q$ by Sylvester's inequality: $\operatorname{rank}\left(X_{1}\right)+\operatorname{rank}\left(X_{2}\right)-n \leq \operatorname{rank}\left(X_{1} X_{2}\right) \leq$
} $\min \left[\operatorname{rank}\left(X_{1}\right), \operatorname{rank}\left(X_{2}\right)\right]$, where $n$ is the number of rows in $X_{2}$. 
Now consider that $\left[\begin{array}{ll}I_{q} & 0\end{array}\right] M=I_{q}$, and post-multiply equation (10) by $\Phi_{t}^{-1}\left(M^{\prime} H_{t}^{-1} M\right)^{-1}$, to obtain equation (9). Positive definiteness follows directly from equation (9) given that $H_{t}$ is positive definite.

Employing Lemmas 1 and 2 we now provide the dynamics of $\Phi_{t}$.

THEOREM 1 (DYNAMICS OF $\Phi_{t}^{-1}$ ). For all $t \in\{s+1, T\}$ we have :

$$
\Phi_{t-1}^{-1}=B_{1}-B_{2}^{\prime}\left(\Phi_{t}^{-1}+B_{3}\right)^{-1} B_{2}, \quad \Phi_{T}^{-1}=M^{\prime} K_{T}^{-1} M
$$

with $\quad B_{1}=M^{\prime} K^{-1} M, \quad B_{2}=M^{\prime} A K^{-1} M, \quad B_{3}=M^{\prime} A K^{-1} A^{\prime} M$.

Proof. Substitute $P_{t}=M \Phi_{t} M^{\prime}$ from Lemma 1 into equation (4):

$$
H_{t-1}=K+A^{\prime} M \Phi_{t} M^{\prime} A, \quad t \leq T .
$$

A standard inversion identity (used later on further occasions) gives: ${ }^{3}$

$$
H_{t-1}^{-1}=K^{-1}-K^{-1} A^{\prime} M\left(\Phi_{t}^{-1}+M^{\prime} A K^{-1} A^{\prime} M\right)^{-1} M^{\prime} A K^{-1} .
$$

${ }^{3}$ Given the matrices $X_{1}, X_{2}, X_{3}$, and $X_{4}$, with $X_{1}$ and $X_{4}$ invertible, we have the identity [Söderström (1994), pp. 156-7]:

$$
\left(X_{1}+X_{2} X_{4}^{-1} X_{3}\right)^{-1}=X_{1}^{-1}-X_{1}^{-1} X_{2}\left(X_{4}+X_{3} X_{1}^{-1} X_{2}\right)^{-1} X_{3} X_{1}^{-1} .
$$


Post-multiplying by $M$ and pre-multiplying by $M^{\prime}$ yields after applying Lemma 2:

$$
\Phi_{t-1}^{-1}=M^{\prime} K^{-1} M-M^{\prime} K^{-1} A^{\prime} M\left(\Phi_{t}^{-1}+M^{\prime} A K^{-1} A^{\prime} M\right)^{-1} M^{\prime} A K^{-1} M,
$$

which is equation (11). $\Phi_{T}^{-1}=M^{\prime} K_{T}^{-1} M$ follows from equation (9) using $H_{T}=K_{T}$ from (3).

By employing the matrix inversion identity of footnote 3 to equation (11), we obtain the following dynamics for $\Phi_{t}$ :

COROllary 1.1 (Dynamics of $\Phi_{t}$ ). For all $t \in\{s+1, T\}$ we have

$$
\Phi_{t-1}=B_{1}^{-1}+B_{1}^{-1} B_{2}^{\prime}\left[\Phi_{t}^{-1}+\left(B_{3}-B_{2} B_{1}^{-1} B_{2}^{\prime}\right)\right]^{-1} B_{2} B_{1}^{-1} .
$$

\subsection{The case of nonsingular $B_{2}$}

Note that the $B_{i}$ matrices in Theorem 1 are all $q \times q$ and that only $B_{2}$ is not symmetric. $B_{I}$ is positive definite and $B_{3}$ is positive semi-definite. By Sylvester's inequality (see footnote 2), $B_{2}$ (= $\left.M^{\prime} A K^{-1} M\right)$ can be of full or less than full rank regardless of whether $A$ has full rank. (However, if $\operatorname{rank}(A)<q$ then $B_{2}$ is certainly singular.) If $B_{2}$ is invertible we obtain additional results; if $B_{2}$ is singular, further reduction is shown to be possible and results analogous to some of those for the case of nonsingular $B_{2}$ are obtained.

COROLLARY 1.2 (REVERSIBILITY). If $B_{2}$ is invertible, the time direction of the recursion for $\left\{\Phi_{t}^{-1}\right\}$ in equation (11) can be reversed: 


$$
\Phi_{t}^{-1}=B_{2}\left(B_{1}-\Phi_{t-1}^{-1}\right)^{-1} B_{2}^{\prime}-B_{3}, \quad t \leq T \text {. }
$$

Thus, using equation (7) in equation (4) to get $H_{t-1}$ in terms of $\Phi_{t}$, the recursion for $\left\{H_{t}\right\}$ is reversible as well, implying that there is a one-to-one correspondence between $H_{t}$ and $H_{T}$ for any t. In contrast the reversibility is not at all clear in the standard Riccati equation (3).

The sequence of reduced Riccati matrices obtained in Theorem 1 can be used with equations (2), (4), and (7) to obtain the sequence $\left\{u_{t}^{\text {opt }}\right\}$ of optimal controls. However, given the transformations employed here there is a more convenient way of calculating the optimal controls when $B_{2}$ has full rank ( $\left.=q\right)$, as in this case the feedback matrix can be shown to be linear in $\Phi_{t}$ :

THEOREM 2 (LINEAR CALCULATION OF FEEDBACK MATRIX). Given $u_{t}^{\text {opt }}=-F_{t} y_{t-1}$, if $\operatorname{rank}\left(B_{2}\right)=q$, then the feedback matrix $F_{t}$ is linear in $\Phi_{t}$ for all $t \leq T-1$ :

$$
F_{t}=-W M \Phi_{t} M^{\prime} A+W K A, \quad t \leq T-1
$$$$
\text { with } \quad W=\left(C^{\prime} C\right)^{-1} C^{\prime} K^{-1}\left[I-A^{\prime} M\left(M^{\prime} K^{-1} A^{\prime} M\right)^{-1} M^{\prime} K^{-1}\right] \text {. }
$$

Proof. From equations (2) and (5) we obtain

$$
C F_{t}=\left(I_{n}-H_{t}^{-1} P_{t}\right) A=\left(I_{n}-H_{t}^{-1} M \Phi_{t} M^{\prime}\right) A, \quad t \leq T,
$$


where the second equality follows from Lemma 1 . To obtain the term $H_{t}^{-1} M$ appearing on the right-hand side of equation (18), we first use equation (13) and the definitions in Theorem 1:

$$
H_{t-1}^{-1} M=K^{-1} M-K^{-1} A^{\prime} M\left(\Phi_{t}^{-1}+B_{3}\right)^{-1} B_{2}, \quad t \leq T .
$$

Use the solution of equation (11) for $\left(\Phi_{t}^{-1}+B_{3}\right)^{-1}$ in equation (19):

$$
H_{t-1}^{-1} M=K^{-1} M-\left(K^{-1} A^{\prime} M\right) B_{2}^{-1^{\prime}}\left(B_{1}-\Phi_{t-1}^{-1}\right), \quad t \leq T .
$$

Update equation (20) by one period (making it valid for $t \leq T-1$ ) and substitute into the right side of (18). Pre-multiplying the left and right sides of equation (18) by $\left(C^{\prime} C\right)^{-1} C^{\prime}$ yields equation (17).

Thus computation of the sequence $\left\{F_{t}\right\}$ of control feedback matrices involves first computing $F_{T}=\left(C^{\prime} K_{T} C\right)^{-1} C^{\prime} K_{T} A$ from equation (2) with $H_{T}=K_{T}$, next iterating equations (11) or (15) to get $\left\{\Phi_{t}\right\}$, and then using equation (17) to obtain the remainder of the feedback matrix sequence.

Comparing to the solution of Problem (1) in equations (2) and (3), the solution proposed here in Theorem 1 [equation (11)] and Theorem 2 [equation (17)] is computationally advantageous. The size of the $\Phi_{t}$ matrix is $q=n-k$, whereas the size of $H_{t}$ is $n$. And the iterations in this approach require fewer matrix multiplications - two in equation (11) and two in equation (17) (aside from the fixed cost of initial matrix calculations) - while the traditional 
approach requires seven multiplications in equation (3) and no additional multiplications in equation (2).

The nonlinear recursive equation (11) for $\Phi_{t}$ can be replaced by a linear equation of larger dimension by using a power method [see for instance Martin and Ammar (1991)]:

THEOREM 3 (LINEAR ITERATION). For any $t \in\{s, T\}$ we have for the case of $\operatorname{rank}\left(B_{2}\right)=q$ :

$$
\Phi_{t}=N_{t} D_{t}^{-1}
$$

with

$$
\text { (22) }\left[\begin{array}{c}
N_{t-1} \\
D_{t-1}
\end{array}\right]=B\left[\begin{array}{c}
N_{t} \\
D_{t}
\end{array}\right], \quad N_{T}=I_{q}, D_{T}=M^{\prime} K_{T}^{-1} M
$$

and hence

$$
\left[\begin{array}{c}
N_{t} \\
D_{t}
\end{array}\right]=(B)^{T-t}\left[\begin{array}{c}
I_{q} \\
M^{\prime} K_{T}^{-1} M
\end{array}\right]
$$

where

$$
\text { (24) } B=\left[\begin{array}{cc}
B_{2}^{-1} B_{3} & B_{2}^{-1} \\
B_{1} B_{2}^{-1} B_{3}-B_{2}^{\prime} & B_{1} B_{2}^{-1}
\end{array}\right] \text {. }
$$

Here the $B_{i}$ are defined in Theorem 1 , and $N_{t}$ and $D_{t}$ are $q \times q$. 
Proof. For $B_{2}$ invertible, we can post-multiply both sides of equation (11) by the inverse of $\left(\Phi_{t}^{-1}+B_{3}\right)^{-1} B_{2}$ and then pre-multiply through by $\Phi_{t-1}$ and post-multiply through by $\Phi_{t}$ to produce:

$$
B_{2}^{-1}-\Phi_{t-1} B_{1} B_{2}^{-1}+B_{2}^{-1} B_{3} \Phi_{t}+\Phi_{t-1}\left(B_{2}^{\prime}-B_{1} B_{2}^{-1} B_{3}\right) \Phi_{t}=0, \quad t \leq T
$$

[Interestingly, the steady-state version of equation (25) (with time subscripts omitted) is of the same form as the algebraic Riccati equation of linear-quadratic control in continuous time (Lancaster and Rodman, 1995)]. Solve equation (25) for $\Phi_{t-1}$ :

$$
\Phi_{t-1}=\left[B_{2}^{-1}+B_{2}^{-1} B_{3} \Phi_{t}\right]\left[B_{1} B_{2}^{-1}+\left(B_{1} B_{2}^{-1} B_{3}-B_{2}^{\prime}\right) \Phi_{t}\right]^{-1}, \quad t \leq T
$$

Postulate $\Phi_{t}=N_{t} D_{t}^{-1}$ (which is true for $t=T$ with $N_{T}=\Gamma, D_{T}=\left(M^{\prime} K_{T}^{-1} M\right) \Gamma$ for arbitrary invertible matrix $\Gamma$, which we set to $I_{q}$ ), use this in equation (26), and post-multiply all four terms by $D_{t}$ to get

$$
\Phi_{t-1}=\left[B_{2}^{-1} D_{t}+B_{2}^{-1} B_{3} N_{t}\right]\left[B_{1} B_{2}^{-1} D_{t}+\left(B_{1} B_{2}^{-1} B_{3}-B_{2}^{\prime}\right) N_{t}\right]^{-1}, \quad t \leq T
$$

Then $N_{t-1}$ and $D_{t-1}$ are the first and second bracketed terms here, confirming equation (22) by induction. Equation (23) follows directly from equation (22). 
To calculate the steady state or "infinite horizon" value, $\Phi_{-\infty} \equiv \lim _{t \rightarrow-\infty} N_{t} D_{t}^{-1}$, one may just iterate equation (22). Note that both $N_{t}$ and $D_{t}$ will generally explode but that $N_{t} D_{t}^{-1}$ remains finite. To prevent computational problems it may be advisable to rescale periodically in finding $\Phi_{-\infty}$ by multiplying both $N_{t}$ and $D_{t}$ by a common scalar, leaving $N_{t} D_{t}^{-1}$ unaffected. The iterations to the steady state can be speeded by using a doubling algorithm, as in Anderson and Moore (1979, pp. 159-160). We successively compute $B^{2},\left(B^{2}\right)^{2}, \ldots . B^{2^{m}}$, rescaling as needed. Then $a\left[\begin{array}{c}N_{T-2^{m}} \\ D_{T-2^{m}}\end{array}\right]=a B^{2^{m}}\left[\begin{array}{c}N_{T} \\ D_{T}\end{array}\right]$, where $a$ is the rescaling factor.

\subsection{Further reduction when $B_{2}$ is singular}

We now consider the case in which $B_{2}$ in equation (11) is singular. This provides the opportunity for further reduction of the size of the Riccati matrix: the $n \times n$ Riccati matrix $H$ has already been reduced to the $q x q$ Riccati matrix $\Phi$; if the $q x q$ matrix $B_{2}$ has rank $r<q$, we can further reduce $\Phi$ to an $r \times r$ matrix to be denoted $\Phi^{*}$.

First put the $q \times q$ matrix $B_{2}$ in standard form:

$$
I_{r}^{q}=Q B_{2} S, \text { where } I_{r}^{q} \equiv\left[\begin{array}{ll}
I_{r} & 0 \\
0 & 0
\end{array}\right], \quad S=\left[\begin{array}{ll}
S_{1} & S_{2} \\
S_{3} & S_{4}
\end{array}\right]
$$

Here $Q$ and $S$ are invertible $q x q$ matrices and $S_{1}$ is $r \times r . \quad S$ must be arranged such that $S_{4}$ is invertible (which may require a row and column rearrangement as discussed in footnote 4). Then we have: 
THEOREM 1*(FURTHER REDUCTION OF $\Phi_{t}$ ). If $B_{2}$ has less than full rank, the dimension of the time-varying core of the Riccati equation, denoted $\Phi_{t}^{*}$, is $r x r$, where $r=\operatorname{rank}\left(B_{2}\right) . \Phi_{t}^{*}$ is positive definite, with $\Phi_{t}^{*-1} \equiv Z^{\prime} \Phi_{t}^{-1} Z$ where $Z$ is defined below, and its dynamics is described by:

$$
\begin{aligned}
& \Phi_{t-1}^{*-1}=B_{1}^{*}-B_{2}{ }^{*}\left(\Phi_{t}^{*-1}+B_{3}^{*}\right)^{-1} B_{2}^{*}, \quad t \leq T-1, \\
& \Phi_{T-1}^{*-1}=Z^{\prime}\left[B_{1}-B_{2}^{\prime}\left(\Phi_{T}^{-1}+B_{3}\right)^{-1} B_{2}\right] Z ;
\end{aligned}
$$

with $B_{1}^{*}=Z^{\prime}\left\{B_{1}-B_{2}^{\prime}\left(B_{1}+B_{3}\right)^{-1} B_{2}+B_{2}^{\prime}\left(B_{1}+B_{3}\right)^{-1}\right.$.

$$
\begin{aligned}
& \left.M^{*}\left[M^{*^{\prime}}\left(B_{1}+B_{3}\right)^{-1} M^{*}\right]^{-1} M^{*^{\prime}}\left(B_{1}+B_{3}\right)^{-1} B_{2}\right\} Z, \\
B_{2}^{*}= & {\left[M^{*^{\prime}}\left(B_{1}+B_{3}\right)^{-1} M^{*}\right]^{-1} M^{*^{\prime}}\left(B_{1}+B_{3}\right)^{-1} B_{2} Z, } \\
B_{3}^{*}= & {\left[M^{*^{\prime}}\left(B_{1}+B_{3}\right)^{-1} M^{*}\right]^{-1}-Z^{\prime} B_{1} Z, }
\end{aligned}
$$

where $Z=\left[\begin{array}{c}I_{r} \\ 0_{q-r, r}\end{array}\right]$ and $M^{*}=\left[\begin{array}{c}I_{r} \\ -S_{4}^{-1^{\prime}} S_{2}^{\prime}\end{array}\right] \quad$ (so $M^{*}$ is $q \times r$ ).

\section{Proof. In Appendix A .}

In equation (27), $B_{2}{ }^{*}$ may or may not be invertible. (Appendix A gives an example in which it is not.) In either case, Theorem $1^{*}$ may be combined with equations (2), (4), (7), and 
(A3) to obtain the sequence of optimal controls based on $\left\{\Phi_{t}^{*}\right\}$. If $B_{2}{ }^{*}$ is not invertible, we can further reduce the dimensionality of the problem by repeatedly applying the reduction process of Theorem $1^{*}$ until the reduced analog of $B_{2}{ }^{*}$ either has zero rank or is invertible. Thus the effective dimension of the original problem is less than or equal to the rank of $B_{2}$.

Note that the proof of Theorem $1 *$ required that the initial condition be stated as of period $T$ - 1. Thus in iterating $H_{t}, H_{T}$ equals $K_{T}$, while for $t \leq T-1 \Phi_{t}^{*}$ is used to compute $\Phi_{t}$, which in turn is used to compute $H_{t}$. Any further reduction shifts back one more period the time of the initial condition for reduced Riccati iteration.

There is no counterpart to Theorem 2 for the case in which any reduction beyond that of Theorem 1 has been applied, because the proof of Theorem 2 rests crucially on invertibility of $B_{2}$ and cannot be generalized to rely on invertibility of $B_{2}{ }^{*}$. In this case of singular $B_{2}$ we can, however, obtain linear iteration analogous to that of Theorem 3 for the nonsingular case:

THEOREM $3^{*}$ (LINEAR ITERATION OF $\left.\Phi_{t}^{*}\right)$. For any $t \in\{s, T\}$ we have for the case of $\operatorname{rank}\left(B_{2}\right)<q$, when Theorem $1 *$ has been applied until $B_{2}^{*}$ is invertible:

$$
\Phi_{t}^{*}=N_{t}^{*} D_{t}^{*-1}
$$

with 
(28) $\left[\begin{array}{c}N_{t-1}^{*} \\ D_{t-1}^{*}\end{array}\right]=B^{*}\left[\begin{array}{c}N_{t}^{*} \\ D_{t}^{*}\end{array}\right], \quad N_{T}^{*}=I_{r}, D_{T}^{*}=Z^{\prime} M^{\prime} K_{T}^{-1} M Z$,

and hence

$$
\left[\begin{array}{c}
N_{t}^{*} \\
D_{t}^{*}
\end{array}\right]=\left(B^{*}\right)^{T-t}\left[\begin{array}{c}
I_{r} \\
Z^{\prime} M^{\prime} K_{T}^{-1} M Z
\end{array}\right]
$$

where

$$
\text { (30) } \quad B^{*}=\left[\begin{array}{cc}
B_{2}^{*-1} B_{3}^{*} & B_{2}^{*-1} \\
B_{1}^{*} B_{2}^{*-1} B_{3}^{*}-B_{2}^{*^{\prime}} & B_{1}^{*} B_{2}^{*-1}
\end{array}\right] \text {. }
$$

The $B_{i}^{*}$ are defined in Theorem $1^{*}$.

\section{Proof. In Appendix B.}

This section has shown how to reduce the size of the dynamic Riccati matrices of optimal control, thereby simplifying computation and revealing the underlying structure of the dynamics. To obtain these results we did not require invertibility either of the matrix of control costs or of the transition matrix $A$. In addition we have provided simple linear dynamics. The usefulness of these results will be demonstrated in the applications section to follow. 


\section{Implications and Applications}

\subsection{Effective dimension of the system}

By Theorem $1^{*}$, the upper bound on the effective dimension of the system (the size of $\Phi_{t}^{*}$ or of $\Phi_{t}$ if $B_{2}$ has full rank) is given by the rank of $B_{2} \equiv M^{\prime} A K^{-1} M$ with $M$ defined in equation (8). This bound may be determined in advance - that is, before theoretical appraisal, estimation, or numerical analysis, or explicit solution of the model. A general indication of the rank of $B_{2}$ is obtained by repeated application of Sylvester's inequality (presented in footnote 2) to the definition of $B_{2}$ given in Theorem 1. Recalling that $n$ represents the dimension of the state vector and $k$ the number of controls, Sylvester's inequality yields:

$$
\operatorname{rank}(A)-2 k \leq \operatorname{rank}\left(B_{2}\right) \leq \min [n-k, \operatorname{rank}(A)] .
$$

Scalar Riccati dynamics will be guaranteed if $n-k$ (the size of $\left.B_{2}\right)=1$ (or, of course, if $\operatorname{rank}(A)$ $=1$ ). This case will be discussed in the next sub-section.

Before we discuss the scalar case, we present a simple example to illustrate the bounds implied by equation (31). Consider a case with $n=3, k=1, C^{\prime}=\left[\begin{array}{lll}0 & 0 & 1\end{array}\right]$, and $K=I_{3}$.

The $3 \times 3$ matrix $A$ is unrestricted. (Note that it is always possible, starting from any like-sized problem with any $C$, to transform the control and state vectors so that $C^{\prime}=\left[\begin{array}{lll}0 & 0 & 1\end{array}\right]$.) Then, $B_{2}$ $=A_{1}$, where $A_{1}$ is the $2 \times 2$ upper left block of $A$. Consequently, there is an infinitude of $A$ matrices for which: (a) $\operatorname{rank}\left(B_{2}\right)=\operatorname{rank}\left(A_{1}\right)=n-k=2$, when the two $1 \times 2$ rows of $A_{1}$ are independent, with $\operatorname{rank}(A)$ equaling either three or two; (b) $\operatorname{rank}\left(B_{2}\right)=\operatorname{rank}\left(A_{1}\right)=$ 
$\operatorname{rank}(A)-2 k=1$, when $A$ has rank one, two, or three and the two $1 \times 2$ rows of $A_{1}$ are dependent; and (c) $\operatorname{rank}\left(B_{2}\right)=\operatorname{rank}\left(A_{1}\right)=\operatorname{rank}(A)-2 k=0$, when all four elements of $A_{1}$ are zero so that $A$ must be singular with either rank one or two.

\subsection{Analytical solution when $\operatorname{rank}\left(B_{2}\right) \leq 1$}

When $B_{2}=M^{\prime} A K^{-1} M$ has rank equal to or less than one, the LQC problem allows

scalar-based analytical solution. When the rank of $B_{2}$ is equal to zero because $B_{2}=0$, Theorem 1 directly shows that $\Phi_{t}$ does not evolve. When the rank of $B_{2}$ is equal to one, Theorem 1 applies if $n-k=1$ (so $B_{2}$ has full rank) and Theorem $1^{*}$ applies if $n-k>1$ (so $B_{2}$ has less than full rank). In what follows we discuss the case $n-k=1$, but if the rank of $B_{2}$ is less than full and equals 1 , Theorem $1^{*}$ applies and the results below all continue to hold if we replace the $B_{i}$ by $B_{i}^{*}$. Equation (11) implies the scalar equation:

$$
\Phi_{t-1}=\left[1+B_{3} \Phi_{t}\right] /\left[B_{1}+\left(B_{1} B_{3}-B_{2}^{2}\right) \Phi_{t}\right], \quad t \leq T
$$

where Theorem 1 defines $B_{1}, B_{2}$, and $B_{3}$, which are scalar in this case (and if Theorem $1^{*}$ applies then $B_{1}^{*}, B_{2}^{*}$, and $B_{3}^{*}$ are scalar).

Mitchell (2000) shows how to find the solution to a scalar equation of the form of equation (32) as follows. Consider first the case of $B_{1} B_{3}-B_{2}^{2} \neq 0$, so that $\Phi_{t}$ evolves nonlinearly (unless $\left.B_{2}=0\right)$. Let $x_{t}=1 /\left(c+\Phi_{t}\right)$ and hence $\Phi_{t}=\left(1-c x_{t}\right) / x_{t}$, where 
$c=\left(B_{1}-B_{3}+r\right) /\left[2\left(B_{1} B_{3}-B_{2}^{2}\right)\right]$ and $r=\left[\left(B_{1}-B_{3}\right)^{2}+4\left(B_{1} B_{3}-B_{2}^{2}\right)\right]^{1 / 2}$. Then use

$\Phi_{t}=\left(1-c x_{t}\right) / x_{t}$ on both sides of equation (32) to obtain a linear equation of evolution for $x_{t}$ :

$$
x_{t-1}=\frac{2\left(B_{1} B_{3}-B_{2}^{2}\right)}{B_{1}+B_{3}+r}+\left(\frac{B_{1}+B_{3}-r}{B_{1}+B_{3}+r}\right) x_{t}, \quad t \leq T,
$$

with solution

$$
x_{t}=\frac{B_{1} B_{3}-B_{2}^{2}}{r}+\left(x_{T}-\frac{B_{1} B_{3}-B_{2}^{2}}{r}\right)\left(\frac{B_{1}+B_{3}-r}{B_{1}+B_{3}+r}\right)^{T-t}, \quad t \leq T \text {. }
$$

Then the solution for $\Phi_{t}$ is found by putting equation (34) into $\Phi_{t}=\left(1-c x_{t}\right) / x_{t}$.

It is also possible for equation (32) to give linear evolution of $\Phi_{t}$. This occurs if and only if $B_{1} B_{3}-B_{2}^{2}=0$. In this linear case the solution of equation (32) for $\Phi_{t}$ is obvious and the eigenvalue is $B_{3} / B_{1}$, which [as Mitchell (2000) shows] may or may not be less than one in magnitude so the linear case may or may not be stabilizable.

To examine the nature of the scalar dynamics, first derive from equation (32):

$$
d \Phi_{t-1} / d \Phi_{t}=B_{2}^{2} /\left[B_{1}+\left(B_{1} B_{3}-B_{2}^{2}\right) \Phi_{t}\right]^{2} \geq 0
$$

Equation (35) suggests several different cases: 
Case 1: $B_{2}=0$. This case is covered equally well by Theorem 1 or Theorem $1 *$. Equation (32) collapses to $\Phi_{t-1}=1 / B_{1}$ which is constant and equal to the endpoint value $\Phi_{T}$ if $K_{T}=K$. Figure 1(a) shows the dynamics of $\Phi_{t}$ and hence $H_{t}$ if $K_{T} \neq K$ so $\Phi_{T} \neq 1 / B_{1}$ : the steady state is reached in one iteration.

Case 2: $B_{2} \neq 0$ and $B_{1} B_{3}-B_{2}^{2} \neq 0$. Note that $B_{1} B_{3}-B_{2}^{2}$ cannot be negative: we know $B_{3}-B_{2} B_{1}^{-1} B_{2}^{\prime}=M^{\prime} A K^{-1}\left[K-M\left(M^{\prime} K^{-1} M\right)^{-1} M^{\prime}\right] K^{-1} A^{\prime} M=M^{\prime} A C\left(C^{\prime} K C\right)^{-1} C^{\prime} A^{\prime} M$, where the last equality follows from substituting equation (9) into equation (7) and the result into equation (5), evaluating the resulting identity at $H_{t}=K$, subtracting $K$ from both sides, and preand post-multiplying both sides by $K^{-1}$. Hence, $B_{3}-B_{2} B_{1}^{-1} B_{2}^{\prime}$ is positive semi-definite, and so in this scalar case multiplying this expression by the positive scalar $B_{1}$ establishes $B_{1} B_{3}-B_{2}^{2} \geq 0$. Then in this case 2 equation (35) implies that $d \Phi_{t-1} / d \Phi_{t}>0$ and $d^{2} \Phi_{t-1} / d \Phi_{t}^{2}<0$; and as $\Phi_{t} \rightarrow \infty$ we have $d \Phi_{t-1} / d \Phi_{t} \rightarrow 0$. Thus, the time path is monotonic and convergent as displayed in Figure 1(b).

Case 3: $B_{2} \neq 0$ and $B_{1} B_{3}-B_{2}^{2}=0$. Now by equation (32), $\Phi_{t-1}=\left(1 / B_{1}\right)+\left(B_{3} / B_{1}\right) \Phi_{t}$ so evolution is linear. This permits the stable case of $B_{3}<B_{1}$ shown in Figure 1(c) (noting that both $B_{1}\left(=M^{\prime} K^{-1} M\right)$, and $B_{3}\left(=M^{\prime} A K^{-1} A^{\prime} M\right)$ must be nonnegative given positive definite $K$ ) as well as the unstable case of $B_{3} \geq B_{1}$, also shown in Figure 1(c).

We have shown here how to solve the case of $n-k=1$ analytically, which was heretofore 
done only for the $n=2, k=1$ case by Mitchell (2000). In addition, we have shown how, due to potential singularities in the transition matrix and its interactions with the cost matrix, other apparently more complex problems can also be solved analytically if the effective dimensionality is 1 .

\subsection{The Amman and Neudecker example}

An example of Amman and Neudecker (1997) can be translated into the framework of equations (1) by moving their control cost matrix into an augmented state cost matrix, augmenting the controls into the state vector, augmenting the transition matrix $A$ with a row and column of zeroes, and augmenting the $C$ matrix with a one to equate identically the new state variable to the control variable. Thus

$$
A=\left[\begin{array}{cccccc}
0.8 & 0 & 0 & 0 & -0.8 & 0 \\
0.8 & 0 & 0 & 0 & -0.8 & 0 \\
0 & 0 & 0 & 0 & 0 & 0 \\
0 & 0 & 0 & 0 & 0 & 0 \\
0.25 & 0 & 0 & 0 & 0 & 0 \\
0 & 0 & 0 & 0 & 0 & 0
\end{array}\right], \quad K=I_{6}, \quad C=\left[\begin{array}{l}
1 \\
0 \\
0 \\
1 \\
0 \\
1
\end{array}\right] .
$$

In this case, the $B_{2}$ matrix can be seen to equal the principal $5 \times 5$ minor of the A matrix, which is the original transition matrix in Amman and Neudecker. The rank of $B_{2}$ is equal to 2; since $B_{2}{ }^{*}$

turns out to have full rank, the effective dimensionality of the problem is 2 .

Theorem $1^{*}$ can be applied to produce the sequence $\left\{\Phi_{t}^{*}\right\}$ and hence $\Phi_{-\infty}^{*}$ and $\Phi_{-\infty}$. Equations (4) and (7) then give $H_{-\infty}$, of which the upper left $5 \times 5$ block is identical to the steady 
state Riccati matrix in Amman and Neudecker (1997). While both our method and that of Amman and Neudecker converge very quickly, ours has the advantage of simultaneously yielding the non-steady-state iterates of the system.

\subsection{Kalman filtering}

The duality between LQC problems and Kalman filtering was first noted by Kalman (1960). Accordingly, our theoretical results apply in the Kalman filtering context as well. Consider the following class of state space models:

$$
\begin{aligned}
& \text { (36a) } y_{\tau-1}=A^{\prime} y_{\tau}+\eta_{\tau}, \quad \eta_{\tau} \sim N(0, K), \\
& \text { (36b) } w_{\tau}=C^{\prime} y_{\tau}, \quad \tau \leq T,
\end{aligned}
$$

where $w_{\tau}$ represents a $k \times 1$ vector of observations and $y_{\tau}$ is an $n \times 1$ vector of unobservable state variables. The interpretation of $\tau$ here is "periods left until the last observation." The disturbances in the state equations are serially uncorrelated and multivariate normal, with covariance matrix $K$. The dimensions of the coefficient matrices are as in the LQC problem. Note that the omission of measurement error in equation (36b) is analogous to the omission of control costs in the LQC model. Measurement error in some of the observations may be incorporated by extending the state vector. The possibility for reducing the dimensionality of the Riccati matrix is quite intuitive here and generalizes the approach of Balvers and Cosimano (1994) in a related case with two state variables and one observation. In their model $w_{1 \tau}=y_{1 \tau}+c y_{2 \tau}$. While the Riccati matrix has dimension $2 \times 2$, containing the variances of $y_{1 \tau}$ and $y_{2 \tau}$ on the diagonal and their covariance off the diagonal, the single scalar $\Phi_{\tau} \equiv P_{1 \tau}$ 
contains all variance-covariance information since, given that the observation is known with certainty, the observation equation implies: $P_{1 \tau}=c^{2} P_{4 \tau}=-c P_{2 \tau}=-c P_{3 \tau}$. This is the intuition for equation (6).

The solution to the Kalman filtering problem modeled in equations (36) consists of a sequence of conditional expectations of the state variables $\left\{E_{\tau} y_{\tau-1}\right\}$ and their conditional covariance matrices $\left\{H_{\tau}\right\}$, for given priors in the initial period $T$. The iterative solution for $\left\{E_{\tau} y_{\tau-1}\right\}$ is [see for instance Harvey (1989)]:

$$
E_{\tau} y_{\tau-1}=A^{\prime} E_{\tau+1} y_{\tau}+F_{\tau}^{\prime} \epsilon_{\tau}, \quad \epsilon_{\tau} \equiv w_{\tau}-C^{\prime} E_{\tau+1} y_{\tau}, \quad \tau \leq T
$$

where $F_{\tau}$ is given in equation (17), which is linear in $\Phi_{\tau}$, with evolution as in equation (11). The sequence $\left\{H_{\tau}\right\}$ is given by equation (3), but may be found more easily from equation (11) with use of equations (4), (7), and (8).

Our reduction results therefore directly apply to problem (36). Accordingly, $\operatorname{rank}\left(B_{2}\right)$ provides an upper bound on the relevant dimension of the problem for solution and (typically maximum likelihood) estimation purposes.

\subsection{A specific Kalman filtering example when $\operatorname{rank}\left(B_{2}\right) \leq 1$}

The example below contains the models in Claar (2000) and Balvers and Wu (2000) as special cases. Claar considers the observed unemployment rate as consisting of the unobserved natural and cyclical unemployment rates. Balvers and $\mathrm{Wu}$ consider observed stock return indices in 18 countries as having a common unobserved world component and an unobserved 
idiosyncratic component. Here, the Riccati matrix would be $19 \times 19$, including all idiosyncratic components plus the common component. But there are 18 observations so that the dimension of $B_{2}$ is unity, and so $\operatorname{rank}\left(B_{2}\right) \leq 1$.

Problem (36) is now specialized to the following coefficient matrices:

$$
\text { (38) } \quad A=\left[\begin{array}{cccc}
a_{0} & 0 & \ldots & 0 \\
0 & a_{1} & \ldots & 0 \\
: & : & & : \\
0 & 0 & \ldots & a_{n-1}
\end{array}\right], \quad K=\left[\begin{array}{cccc}
k_{0} & 0 & \ldots & 0 \\
0 & k_{1} & \ldots & 0 \\
: & : & & : \\
0 & 0 & \ldots & k_{n-1}
\end{array}\right], \quad C=\left[\begin{array}{ccc}
1 & \ldots & 1 \\
-- & -- & -- \\
1 & \ldots & 0 \\
: & \ddots & : \\
0 & \ldots & 1
\end{array}\right] \text {. }
$$

Note that both $A$ and $K$ are $n \times n$, whereas $C$ is $n x(n-1)$. Thus, for the Balvers and Wu example the first row of each of the coefficient matrices refers to the "common" component; there are $n$-1 idiosyncratic components, one for each observation. For the Claar example, $n=2$ and so $C=\left[\begin{array}{l}1 \\ 1\end{array}\right]$.

From equation (8) we can obtain the $M$ matrix with dimension $n \times 1$ :

(39) $\quad M=\left[\begin{array}{lllll}1 & -1 & -1 & \ldots & -1\end{array}\right]^{\prime}$.

From equations (38) and (39) we straightforwardly calculate the scalar coefficients of the $\Phi_{\tau}$ recursion of equation (11): 


$$
B_{1}=\sum_{i=0}^{n-1} k_{i}^{-1}, \quad B_{2}=\sum_{i=0}^{n-1} a_{i} k_{i}^{-1}, \quad B_{3}=\sum_{i=0}^{n-1} a_{i}^{2} k_{i}^{-1} .
$$

The cases of Figures 1(a) - 1(c) all arise here. For brevity we state here only that Case 3 of Figure 1(c) can be ruled out except for the uninteresting case where all coefficients are equal. Case 1 of Figure 1(a) occurs if $B_{2}=0$. In this case, all there is to be learned is completed in one period. Otherwise, we have Case 2 in Figure 1(b).

To illustrate Theorem 2, we find the Kalman gain matrix $F_{\tau}^{\prime}$ from equation (17). Simple but tedious calculation shows that there are three basically different forms of the Kalman gain coefficients $F_{j i \tau}^{\prime}=F_{i j \tau}$ :

$$
F_{i j \tau}=\frac{\partial E_{\tau+1} x_{j-1 \tau}}{\partial \epsilon_{i \tau+1}}=\left\{\begin{array}{l}
-a_{j-1} z_{i \tau}, \quad j \neq 1, \quad j \neq i+1 \\
+a_{0} z_{i \tau}, \quad j=1 \\
-a_{i}\left(z_{i \tau}-1\right), \quad j=i+1,
\end{array}\right.
$$

where $\quad z_{i \tau}=\frac{a_{i} k_{i}^{-1}}{B_{2}}+\Phi_{\tau} k_{i}^{-1}\left(1-\frac{a_{i} B_{1}}{B_{2}}\right)$.

It can be shown that, for proper priors, $z_{i \tau}$ is always positive in non-trivial cases. Thus, equation (41) implies that a surprise increase in observation $w_{i \tau+1}$ causes an increase in the expected value of next period's common component $y_{0 \tau}$ and thus a decrease in the expected value of next period's idiosyncratic components $y_{j-1, \tau}$ unless $j=i+1$. In this case, $y_{i \tau}$, 
representing the idiosyncratic component in the observation $i$ itself, may decrease when uncertainty $\Phi_{\tau}$ is high, but eventually increases as $\Phi_{\tau}$ reaches its steady state. The intuition is that, with high uncertainty, the estimate of the common component $y_{0 \tau}$ may be revised substantially upwards so that the estimate of the idiosyncratic component $y_{i \tau}$ could fall even though the observation $w_{i \tau}=y_{0 \tau}+y_{i \tau}$ increases.

\section{Summary and Conclusion}

This paper presents a procedure for simplifying and solving LQC models. The procedure can be summarized in the following algorithm:

Step 1. If necessary, transform the LQC problem to fit the structure of equations (1).

Step 2. First obtain $M$ from equation (8) and subsequently obtain $B_{1}, B_{2}, B_{3}$, and $\Phi_{T}^{-1}$ as given in Theorem 1.

Step 3. If $B_{2}$ has full rank find $\left\{\Phi_{t}\right\}$ from Theorem 1 (or Theorem 3). Then skip to Step 8 .

Step 4. If $B_{2}$ does not have full rank, find the $S$ matrix by transforming $B_{2}$ into standard form and extract $S_{4}^{-1}$ and $S_{2}$. (If $S_{4}^{-1}$ does not exist consider footnote 4.)

Step 5. Obtain $M^{*}, B_{1}{ }^{*}, B_{2}{ }^{*}, B_{3}{ }^{*}$, and $\Phi_{T-1}^{*-1}$ as given in Theorem $1^{*}$. If $B_{2}{ }^{*}$ is not invertible repeat Steps 4 and 5 .

Step 6. Find $\left\{\Phi_{t}^{*-1}\right\}$ from Theorem $1^{*}$ or $3^{*}$.

Step 7. Employ equation (A3) to deduce $\left\{\Phi_{t}\right\}$ from $\left\{\Phi_{t}^{*-1}\right\}$. 
Step 8. Substitute $\left\{\Phi_{t}\right\}$ into equation (7) to find $\left\{P_{t}\right\}$ and then use equation (4) to generate $\left\{H_{t}\right\}$, if $\left\{P_{t}\right\}$ and $\left\{H_{t}\right\}$ are needed. The end matrix $H_{T}$ is generated as $H_{T}=K_{T}$, and $H_{T-1}$ is generated from $\Phi_{T}$ via equations (4) and (7).

Step 9. If $B_{2}$ has full rank, find the feedback matrix sequence $\left\{F_{t}\right\}$ from equation (2), or, for $t \leq T-1$, from Theorem 2. In the LQC problem, the optimal control vector $u_{t}^{o p t}$ equals $-F_{t} y_{t-1}$. In the Kalman problem $F_{t}^{\prime}$ is the information filter appearing in equation (37).

Step 9'. If $B_{2}$ is singular, use $\left\{H_{t}\right\}$ from Step 8 and use equation (2) to find the $\left\{F_{t}\right\}$ matrix sequence.

This procedure has a series of advantages compared to traditional procedures for solving the finite horizon and infinite horizon LQC problem, such as Vaughan (1970), and compared to more recent procedures. First, it provides a simple calculation (the rank of $B_{2}$ ) to establish an upper bound on the effective dimension of the problem. It is then possible to find in advance, without computing the solution, how complicated or simple the dynamics is or how much computing time may be saved by reformulating the problem. Second, the procedure leads to scalar-based analytical solutions when the rank of $B_{2}$ is zero or one, and to matrix size reductions in other cases when the transition matrix and the matrix of control costs may be less than full rank. Third, in the general $n x k$ problem, iterations can be conducted with matrices that have the size of the effective dimension of the problem, which, especially for large finite-horizon problems, may lead to substantially speedier computation. Fourth, rather than solving the problem of singular transition and control-cost matrices by increasing the dimension of the state vector as is the common approach in recent work, our procedure reduces the dimensionality. Fifth, no eigenvalue computations or Jordan or Schur decompositions, with possible accompanying 
numerical instabilities, are required for our approach.

Aside from procedural advantages, the results in the paper provide direct analytical insights. For instance, the fact in Theorem 2 that, when $B_{2}$ has full rank, the optimal feedback matrix can be obtained as a linear transformation of the dynamic core of the Riccati matrix may be valuable in theoretical applications. Some of the results developed in this paper appear to be quite novel from an applied mathematical perspective and may turn out to be of use in establishing further results and in other applications. For instance, the rank of the $B_{2}$ matrix may be helpful in dealing with identification problems in the Kalman context. Or, some of the results may be applicable to other issues in least squares-based time-series econometrics. 


\section{Appendix A: Proof of Theorem 1*}

Consider a generalized inverse of $B_{2}$ given as: $B_{2}{ }^{I}=S I_{r}^{q} Q$. It is easy to check that $B_{2}{ }^{I}$ is indeed a generalized inverse of $B_{2}$ since $B_{2} B_{2}{ }^{I} B_{2}=Q^{-1}\left(I_{r}^{q}\right)^{3} S^{-1}=B_{2}$ and since $I_{r}^{q}$ is an idempotent matrix. Define $\Theta=\left(I_{q}-B_{2}{ }^{I} B_{2}\right) S\left[\begin{array}{c}0_{r, q-r} \\ I_{q-r}\end{array}\right]$. Then by design $B_{2} \Theta=0_{q, q-r}$ and straightforward multiplication shows that:

$$
\Theta=\left[I_{q}-S\left(I_{r}^{q}\right)^{2} S^{-1}\right] S\left[\begin{array}{c}
0_{r, q-r} \\
I_{q-r}
\end{array}\right]=S\left(I_{q}-I_{r}^{q}\right)\left[\begin{array}{c}
0_{r, q-r} \\
I_{q-r}
\end{array}\right]=\left[\begin{array}{c}
S_{2} \\
S_{4}
\end{array}\right] .
$$

Since $B_{2} \Theta=0$ it follows from equation (11) updated one period that:

$$
\left(\Phi_{t}^{-1}-B_{1}\right) \Theta=\left(\Phi_{t}^{-1}-B_{1}\right)\left[\begin{array}{c}
S_{2} \\
S_{4}
\end{array}\right]=0_{q, q-r}, \quad t \leq T-1
$$

Partition $\Phi_{t}^{-1}$ and $B_{1}$ according to $S$ and extract the $r x r$ upper left block of $\Phi_{t}^{-1}$ as $Z^{\prime} \Phi_{t}^{-1} Z$.

Take $S_{4}$ as invertible ${ }^{4}$ and solve equations (A2) similarly to equation (6). This yields:

${ }^{4}$ If $S_{4}$ is not invertible we can always rearrange the rows of $S$ by pre-multiplying by some permutation matrix $G$ such that $\hat{S} \equiv G S$ with $\hat{S}_{4}$ invertible. In this case transform all $B_{i}$ to obtain $G^{\prime} B_{i} G$. In order for equation (11) to continue to hold we must also transform $\Phi_{t}^{-1}$ to $G^{\prime} \Phi_{t}^{-1} G$ and in order for the procedure to work we must also set $\hat{Q} \equiv Q G^{\prime}$. Then we can proceed as above. We then obtain $G^{\prime}\left(\Phi_{t}^{-1}-B_{1}\right) G$ in equation (A3) but can recover $\Phi_{t}^{-1}$ by inverting the known $G$ matrix. 


$$
\Phi_{t}^{-1}-B_{1}=M^{*}\left(\Phi_{t}^{*-1}-B_{11}\right) M^{*^{\prime}}, \quad M^{*}=\left[\begin{array}{c}
I_{r} \\
-S_{4}^{-1^{\prime}} S_{2}^{\prime}
\end{array}\right], \quad t \leq T-1,
$$

where $\Phi_{t}^{*-1} \equiv Z^{\prime} \Phi_{t}^{-1} Z$ represents the upper left block of $\Phi_{t}^{-1}$ and $B_{11} \equiv Z^{\prime} B_{1} Z$

represents the upper left block of $B_{1}$. As $\Phi_{t}^{-1}$ and $B_{1}$ are positive definite, so are $\Phi_{t}^{*-1}$ and $B_{11}$.

To obtain the dynamics of $\Phi_{t}^{*-1} \equiv Z^{\prime} \Phi_{t}^{-1} Z$, post-multiply equation (11) by $Z$ and premultiply by $Z^{\prime}$, and use equation (A3) on the right side to produce:

$$
\Phi_{t-1}^{*-1}-B_{11}=-Z^{\prime} B_{2}^{\prime}\left[M^{*}\left(\Phi_{t}^{*-1}-B_{11}\right) M^{*^{\prime}}+\left(B_{1}+B_{3}\right)\right]^{-1} B_{2} Z, \quad t \leq T-1 .
$$

To manipulate equation (A4), consider that the term in brackets is positive definite (as it equals the sum of a positive definite matrix $\Phi_{t}^{-1}$ and a positive semi-definite matrix $B_{3}$ ). Further, $B_{2} Z$ has full column rank $r: \quad B_{2} Z=Q^{-1} I_{r}^{q} S^{-1} Z, \quad S^{-1} \equiv\left[\begin{array}{cc}\Sigma_{1} & \Sigma_{2} \\ \Sigma_{3} & \Sigma_{4}\end{array}\right]$. Thus $I_{r}^{q} S^{-1} Z=\left[\begin{array}{c}\Sigma_{1} \\ 0\end{array}\right]$, where $\operatorname{rank}\left(\left[\begin{array}{c}\Sigma_{1} \\ 0\end{array}\right]\right)=\operatorname{rank}\left(\Sigma_{1}\right), \operatorname{so} \operatorname{rank}\left(B_{2} Z\right)=\operatorname{rank}\left(\Sigma_{1}\right)$. From footnote 4 we can assume without loss of generality that $S_{4}$ has full rank $q-r$. We know that $\Sigma_{1}=\left(S_{1}-S_{2} S_{4}^{-1} S_{3}\right)^{-1}$ [Anderson and Moore (1990), p. 349] because $S_{4}^{-1}$ exists and because 
$\left|S_{4}\right| \cdot\left|S_{1}-S_{2} S_{4}^{-1} S_{3}\right|=|S| \neq 0$ so $\left|S_{1}-S_{2} S_{4}^{-1} S_{3}\right| \neq 0$ [Söderström (1994), p. 162]. Thus $\Sigma_{1}$ has full rank $r$, so that $B_{2} Z$ has full column rank. This fact, together with the positive definiteness of the term in brackets, establishes that the left-hand side of equation (A4) is negative definite and thus $\Phi_{t}^{*-1}-B_{11}$ is invertible. We can now use the inversion identity (see footnote 3) to rewrite the term in brackets, since the relevant inverses exist:

$$
\begin{aligned}
\Phi_{t-1}^{*-1}= & Z^{\prime}\left[B_{1}-B_{2}^{\prime}\left(B_{1}+B_{3}\right)^{-1} B_{2}\right] Z+Z^{\prime} B_{2}^{\prime}\left(B_{1}+B_{3}\right)^{-1} M^{*} . \\
& \quad\left[M^{*^{\prime}}\left(B_{1}+B_{3}\right)^{-1} M^{*}+\left(\Phi_{t}^{*-1}-B_{11}\right)^{-1}\right]^{-1} M^{*^{\prime}}\left(B_{1}+B_{3}\right)^{-1} B_{2} Z, \quad t \leq T-1 .
\end{aligned}
$$

Again use the inversion identity to reformulate the second expression in brackets:

$$
\begin{gathered}
\Phi_{t-1}^{*-1}=Z^{\prime}\left[B_{1}-B_{2}^{\prime}\left(B_{1}+B_{3}\right)^{-1} B_{2}\right] Z+Z^{\prime} B_{2}^{\prime}\left(B_{1}+B_{3}\right)^{-1} M^{*}\left\{\left[M^{*^{\prime}}\left(B_{1}+B_{3}\right)^{-1} M^{*}\right]^{-1}\right. \\
-\left[M^{*^{\prime}}\left(B_{1}+B_{3}\right)^{-1} M^{*}\right]^{-1}\left\{\Phi_{t}^{*-1}-Z^{\prime} B_{1} Z+\left[M^{*^{\prime}}\left(B_{1}+B_{3}\right)^{-1} M^{*}\right]^{-1}\right\}^{-1} . \\
\left.\left[M^{*^{\prime}}\left(B_{1}+B_{3}\right)^{-1} M^{*}\right]^{-1}\right\} M^{*^{\prime}}\left(B_{1}+B_{3}\right)^{-1} B_{2} Z, \quad t \leq T-1 .
\end{gathered}
$$

Note that the inverse of the term in small braces exists by the invertibility of the second term in brackets in equation (A5), since, when $\left[X+Y^{-1}\right]^{-1}$ exists, so does $\left[Y+X^{-1}\right]^{-1}$ with $Y \equiv \Phi_{t}^{*-1}-B_{11}$ and $X \equiv M^{*^{\prime}}\left(B_{1}+B_{3}\right)^{-1} M^{*}$ both invertible.

Equation (A6) directly yields equation (27) in Theorem 1*. The initial condition $\Phi_{T-1}^{*-1}$ is obtained from using equation (11) in $\Phi_{T-1}^{*-1} \equiv Z^{\prime} \Phi_{T-1}^{-1} Z$. 
An example in which $B_{2}{ }^{*}$ is singular (after a single application of the reduction in Theorem

$\left.1^{*}\right)$ is as follows:

$$
K=I_{4}, \quad C=\left(\begin{array}{c}
0_{2,2} \\
I_{2}
\end{array}\right), \quad A=\left(\begin{array}{ll}
A_{1} & A_{2} \\
A_{3} & A_{4}
\end{array}\right), \quad A_{1}=\left(\begin{array}{cc}
1 & 2 \\
-0.5 & -1
\end{array}\right), \quad A_{2}=\left(\begin{array}{ll}
0 & 0 \\
0 & 0
\end{array}\right) .
$$

Then $B_{2}=A_{1}$ and so $\operatorname{rank}\left(B_{2}\right)=1$ and hence $B_{2}^{*}$ is a scalar. Then

$Q=\left(\begin{array}{cc}1 & 0 \\ -2 & 1\end{array}\right), S=\left(\begin{array}{cc}1 & -0.5 \\ 0 & -1\end{array}\right)$, so $M^{*}=\left(\begin{array}{c}1 \\ -0.5\end{array}\right)$. Therefore $M^{*^{\prime}}\left(B_{1}+B_{3}\right)^{-1} B_{2} Z=0$

so that $B_{2}^{*}=0$; hence $\operatorname{rank} B_{2}^{*}=0<1$.

\section{Appendix B: Proof of Theorem 3*}

First note that $B^{*}$ exists since $B_{2}{ }^{*}$ is invertible after repeated application of Theorem $1^{*}$. Note

that since $B_{2}{ }^{*}$ is invertible we can apply to equation (27) the same transformations that were

applied to equation (11) in obtaining equation (25), and the result can be solved out to infer:

$$
\Phi_{t-1}^{*}=\left[B_{2}^{*-1}+B_{2}^{*-1} B_{3}^{*} \Phi_{t}^{*}\right]\left[B_{1}^{*} B_{2}^{*-1}+\left(B_{1}^{*} B_{2}^{*-1} B_{3}^{*}-B_{2}^{*^{\prime}}\right) \Phi_{t}^{*}\right]^{-1}, \quad t \leq T .
$$

Then the proof proceeds as the proof of Theorem 3, with $N_{T}^{*}=\Gamma$ and $D_{T}^{*}=Z^{\prime} M^{\prime} K_{T}^{-1} M Z \Gamma$

for arbitrary invertible matrix $\Gamma$ (as we know by applying $\Phi_{t}^{*-1} \equiv Z^{\prime} \Phi_{t}^{-1} Z$ with

$\left.\Phi_{T}^{-1}=M^{\prime} K_{T}^{-1} M\right)$ and with all $\Phi_{t}$ and $B_{i}$ having asterisks attached. 


\section{References}

Amman, H. M., 1997, Numerical methods for linear-quadratic models, in H.M. Amman, D.A. Kendrick, and J. Rust, eds., Handbook of Computational Economics (North-Holland, Amsterdam).

Amman, H. M. and H. Neudecker, 1997. Numerical solutions of the algebraic matrix Riccati equation, Journal of Economic Dynamics and Control 21, 363-369.

Anderson, B. D. A. and J. B. Moore, 1979. Optimal filtering (Prentice Hall, Englewood Cliffs).

Anderson, B. D. A. and J. B. Moore, 1990. Optimal control: linear quadratic methods (Prentice Hall, Englewood Cliffs).

Anderson, E., L. P. Hansen, E. R. McGrattan and T. Sargent, 1997. Mechanics of forming and estimating dynamical linear economies, in H.M. Amman, D.A. Kendrick, and J. Rust, eds., Handbook of Computational Economics (North-Holland, Amsterdam).

Anderson, G. and G. Moore, 1985. A linear algebraic procedure for solving linear perfect foresight models, Economics Letters 17, 247-252.

Balvers, R. J. and T. F. Cosimano, 1994. Inflation variability and gradualist monetary policy, Review of Economic Studies 61, 721-738.

Balvers, R. J. and Y. Wu, 2000. Momentum and mean reversion across national equity markets, Working paper, Rutgers University.

Binder, M. and M. H. Pesaran, 2000. Solution of finite-horizon multivariate linear rational expectations models and sparse linear systems, Journal of Economic Dynamics and Control 24, 325-346.

Blanchard, O. J. and Kahn, C. M., 1980. The solution of linear difference models under rational expectations, Econometrica 48, 1305-11.

Chow, G. C., 1975. Analysis and control of dynamic economic systems (Wiley, New York).

Claar, V. V., 2000. The natural rate of unemployment, its estimation, and evaluation of its estimators, Ph. D. dissertation, West Virginia University.

Ehlgen, J., 1999. A nonrecursive solution method for the linear-quadratic optimal control problem with a singular transition matrix, Computational Economics 13, 17-23. 
Hannan, E. J. and M. Deistler, 1988. The statistical theory of linear systems (John Wiley and Sons, New York).

Harvey, A. W., 1989. Forecasting, structural time series models and the Kalman filter (Cambridge University Press, New York).

Kalman, R. E., 1960. A new approach to linear filtering and prediction problems, Journal of Basic Engineering, 35-45.

King, R. G. and M. Watson, 1997. System reduction and solution algorithms for singular linear difference systems under rational expectations, Working paper, Princeton University.

King, R. G. and M. Watson, 1998. The solution of singular linear-difference systems under rational expectations, International Economic Review 39, 1015-27.

Klein, P., 2000. Using the generalized Schur form to solve a multivariate linear rational expectations model, Journal of Economic Dynamics and Control 24, 1405-24.

Lancaster, P. and L. Rodman, 1995. Algebraic Riccati Equations (Clarendon Press, Oxford).

Ljungqvist, L. and T. J. Sargent, 2000. Recursive macroeconomic theory (MIT Press, Cambridge).

Martin, C. F., and G. Ammar, 1991. The geometry of the matrix Riccati equation and associated eigenvalue method, in Bittani, Laub, and Willems, eds., The Riccati equation (Springer Verlag, New York).

Mitchell, D.W., 2000. An analytic Riccati solution for two-target discrete-time control, Journal of Economic Dynamics and Control 24, 615-622.

Sims, C. S., 2000, Solving linear rational expectations models, Working paper, Yale University.

Söderström, T., 1994. Discrete-time stochastic systems: estimation and control (Prentice-Hall, New York).

Vaughan, D.R., 1970. A nonrecursive algebraic solution for the discrete Riccati equation, IEEE Transactions on Automatic Control 15, 597-599. 


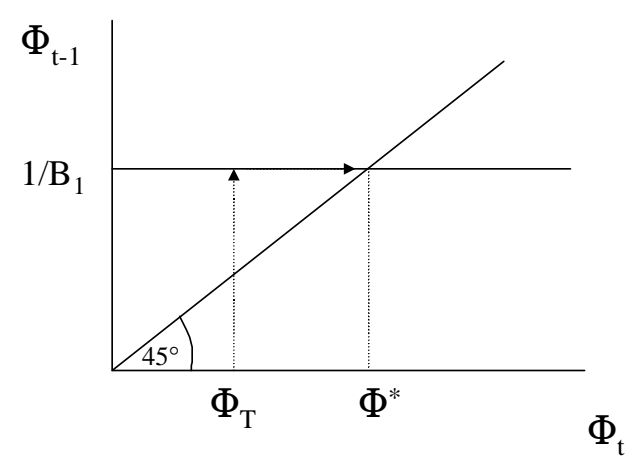

Fig. 1(a): $B_{2}=0$

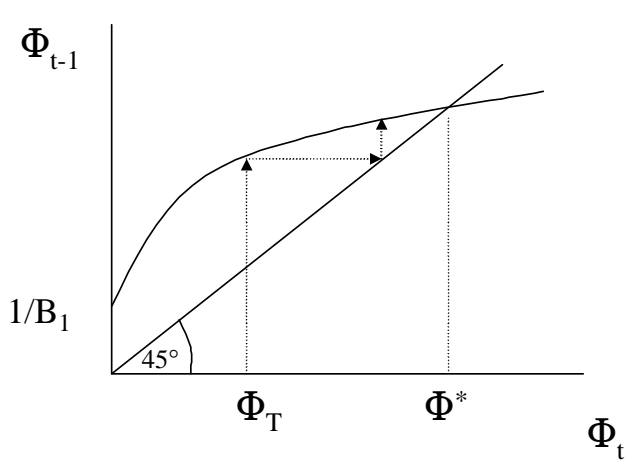

Fig. 1(b): $B_{2} \pi 0, \quad B_{1} B_{3}>B_{2}^{2}$

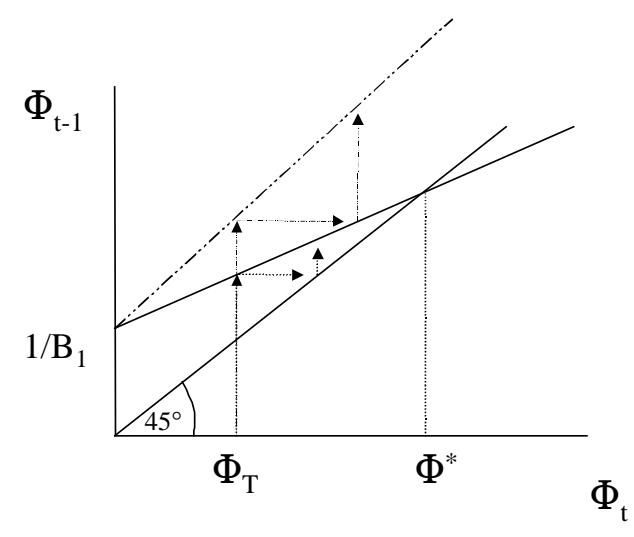

Fig. 1(c): $B_{2} \pi 0, \quad B_{1} B_{3}=B_{2}^{2}$ 